\title{
Tougu Xiaotong capsule inhibits the tidemark replication and cartilage degradation of papain-induced osteoarthritis by the regulation of chondrocyte autophagy
}

\author{
XIHAI LI ${ }^{1}$, WENNA LANG ${ }^{2}$, HONGZHI YE ${ }^{1}$, FANGRONG YU ${ }^{3},{\text { HUITING } \text { LI }^{3} \text {, JIASHOU CHEN }}^{3}$, \\ LIANGLIANG CAI ${ }^{3}$, WENLIE CHEN ${ }^{1}$, RUHUI LIN ${ }^{3}$, YUNMEI HUANG ${ }^{3}$ and XIANXIANG LIU ${ }^{1}$
}

\begin{abstract}
${ }^{1}$ Academy of Integrative Medicine, Fujian University of Traditional Chinese Medicine, Fuzhou; ${ }^{2}$ Research Base of Traditional Chinese Medicine Syndrome, Fujian University of Traditional Chinese Medicine, Fuzhou; ${ }^{3}$ Fujian Key Laboratory of Integrative Medicine on Geriatrics, Fujian University of Traditional Chinese Medicine, Fuzhou, Fujian 350122, P.R. China
\end{abstract}

Received January 6, 2013; Accepted March 26, 2013

DOI: $10.3892 / \mathrm{ijmm} .2013 .1341$

\begin{abstract}
The tidemark is located between calcified and non-calcified cartilage matrices. Tidemark replication plays an important role in the pathogenesis of osteoarthrosis (OA). Autophagy, or cellular self-digestion, is an essential cellular homeostasis mechanism that was found to be deficient in osteoarthritic cartilage. This study evaluated the effects of Tougu Xiaotong capsule (TXC) on the tidemark replication and cartilage degradation, and also investigated LC3 I/II, which executes autophagy, the potential role of ULK1, an inducer of autophagy, and Beclin1, a regulator of autophagy, in the development of a papain-induced OA in rat knee joints. Using a papain-injected knee rat model, standard histological methods were used to validate our model as well as treatment with TXC or glucosamine $(\mathrm{GlcN})$. After 12 weeks of treatment, the changes of cartilage structure were observed by digital radiography (DR), optical microscopy, scanning electron microscopy and transmission electron microscopy, and the LC3 I/II, ULK1 and Beclin1 levels were measured by western blotting. Cartilage degradation was evaluated by the Mankin score on paraffin-embedded sections stained with Safranin O-fast green. TXC was found to improve the arrangement of subchondral bone collagen fibers and calcium phosphate crystals, inhibit the tidemark replication and delay the cartilage degradation in the papain-induced OA. Our results also showed that LC3 I/II, ULK1 and Beclin1 levels in both the TXC+OA and GlcN+OA groups were significantly increased compared to those in the
\end{abstract}

Correspondence to: Dr Xianxiang Liu, Academy of Integrative Medicine, Fujian University of Traditional Chinese Medicine, 1 Huatuo, University Town, Minhou Shangjie, Fuzhou, Fujian 350122, P.R. China

E-mail: liuxianxiang@163.com

Key words: osteoarthritis, tidemark, autophagy, subchondral bone, Tougu Xiaotong capsule
OA group. The results indicate that TXC could inhibit the tidemark replication and cartilage degradation by the regulation of chondrocyte autophagy.

\section{Introduction}

Osteoarthritis (OA), a highly prevalent, slowly progressive, degenerative disease of diarthrodial joints, is characterized by a progressive degradation of articular cartilage associated with marginal osteophyte formation, progressive symptomatic loss of mechanical function and remodeling of the subchondral bone, belonging to the GU BI of Traditional Chinese Medicine (TCM) $(1,2)$. As the precise molecular mechanism of OA has yet to be fully elucidated, a wide variety of animal models have been developed to study osteoarthritic progression, characterize the features of the early pathological changes of OA and to evaluate new drugs and/or original therapies (3). The papain-induced OA model has been widely studied in various animal species such as rats, thus providing new insights into pathogenic mechanisms and impact of hyaline cartilage $(4,5)$. In this model, there is a sequence of events in which the degradation of the superficial zone develops into fibrillations of the cartilage and eventually leads to ulcerations, erosions and tidemark replication.

Tidemark, at which non-mineralized cartilage comes to contain hydroxyapatite, is a chondro-osseous junction between cartilage and bone in diarthrodial joints (6). The vicinity of the tidemark enhanced metabolic activity, consistent with mineralization, including the expression of alkaline phosphatase, the deposition of type $\mathrm{X}$ collagen and the ability to bind tetracycline in vivo. The tidemark replication is considered a characteristic of the osteoarthrotic process with the advance of a calcification front advancing into the non-calcified cartilage of zone IV (7). The changes of tidemark are considered to be coordinated with the resorption of the calcified cartilage of zone $\mathrm{V}$, as the subchondral bone thickens and replaces it (8).

The loss of chondrocyte function was found to be a persistent and important event in OA. A variety of stimuli, such as mechanical injury, loss of growth factors or excessive reactive oxygen species, can induce chondrocyte depletion (9). 
Since chondrocyte is solely responsible for the maintenance and production of extracellular matrix (ECM), chondrocyte depletion is indicated in the cartilage degradation, which pertains to OA pathogenesis $(10,11)$. Chondrocyte apoptosis was thought to be a major cause of chondrocyte depletion during OA progression, so enhanced chondrocyte apoptosis is considered to be a sign of progressive cartilage degradation. However, the extent of the contribution of apoptotic cell death to chondrocyte depletion in OA progression remains to be resolved. Previous studies reported that another type of cell death, autophagy, may be involved in chondrocyte death during OA progression $(12,13)$.

Tougu Xiaotong capsule (TXC), a TCM formulation, consists of a combination of four natural products including Radix Morindae Officinalis, Radix Paeoniae Alba, Rhizoma Chuanxiong and Glabrous Sarcandra Herb. These natural products together confer TXC properties of nourishing Shen, supplementing Jing, filling in Sui, stretching tenders and dredging collaterals to strengthen tendons and bones at the theories of TCM. TXC has been used for the osteoarthritic treatment in the Second People's Hospital Affiliated to Fujian University of TCM for two decades, and it has been shown to have significant therapeutic effects on OA in the clinical trials, such as evident improvements in osteoarthritic symptoms, pain, swelling and motion of joint. Previously, we reported that TXC could inhibit chondrocyte apoptosis by upregulation of Bcl-2, downregulation of p53, caspase-9 and caspase-3 (14). However, the molecular mechanism of the therapeutic effect of TXC remains largely unknown. Therefore, using a papaininduced OA in rat knee joints, we evaluated the effect of TXC on the tidemark replication and cartilage degradation, and investigated the underlying mechanisms of TXC in the regulation of chondrocyte autophagy.

\section{Materials and methods}

Animals. Forty 4-week-old male Sprague-Dawley (SD) rats of Specific Pathogen Free (SPF), qualified number SCXK (Shanghai) 2007-0005, were purchased from the Shanghai Slack Laboratory Animal Co. (Shanghai, China). The Fujian University of TCM Experimental Animal Centre offers SPF medical laboratory animal environmental facilities, qualified number SYXK(Min) 2009-0001. The care and use of the laboratory animals complied with the Guidance Suggestions for the Care and Use of Laboratory Animals 2006 of the Ministry of Science and Technology, China.

Experimental design. After one week of acclimation, the rats received a $12 \mu \mathrm{l}$ intra-articular injection of a $1 \mathrm{U} / \mathrm{ml}$ of L-cysteine-activated papain in phosphate buffered saline (PBS) (Sigma, St. Louis, MO, USA) in their double knee joints at 1, 4 and 7 days (4). Eight weeks after papain-induced $\mathrm{OA}$, the animals were randomly divided into four groups. The TXC+OA group received oral TXC (the Second People's Hospital Affiliated to Fujian University of TCM, medical license no. MINZHIZI Z20100006; $184 \mathrm{mg} / \mathrm{kg} / \mathrm{day})$. The glucosamine $(\mathrm{GlcN})+\mathrm{OA}$ group received oral GlcN sulfate (Sigma; $150 \mathrm{mg} / \mathrm{kg} /$ day). The OA group and control group (non papain-induced OA) received equivalent saline only. All groups were treated once a day for 12 consecutive weeks, following which the animals were sacrificed. The changes of cartilage structure and tidemark were observed by digital radiography (DR), optical microscopy, scanning electron microscopy (SEM) and transmission electron microscopy (TEM).

Gross morphology of the knee joints. The gross morphological changes in cartilage were examined by DR (LDRD-01BL, Beijing Aerospace Zhongxing Medical System Co., Ltd., China), and the grade of knee joint degradation of DR films was according to the Kellgren-Lawrence X-ray grade standard (15).

The tibial plateau cartilage was bivalved in the coronal plane with a sharp osteotome. The exposed surface was rinsed with PBS repeatedly to remove blood and bone marrow. Specimens, $5 \mathrm{~mm}$ × $5 \mathrm{~mm}$ x $5 \mathrm{~mm}$ in size for SEM, were then fixed with $2 \%$ glutaraldehyde solution, washed with $0.1 \mathrm{M}$ sodium cacodylate buffer, and post-fixed with $1 \%$ osmium tetroxide. After dehydrating with an alcohol gradient series, and dehydrating with isoamyl acetate again, the specimen was dried using a critical point dryer with HCP-2. After coating with a layer of gold, all specimens were observed under SEM (JSM-6380LV, JEOL, Japan).

Histopathological examination of the knee joints. The joints were sectioned $0.5 \mathrm{~cm}$ above and below the joint line, fixed in $10 \%$ neutral buffered formalin for 3 days, and then decalcified for 2 weeks in buffered $12.5 \%$ ethylenediaminetetraacetic acid (EDTA) and formalin solution. The cartilage was stained with Safranin O-fast green stains to assess the general morphology and matrix proteoglycans. Cartilage histological changes were evaluated according to the Mankin score (16).

Ultrastructural examination of the knee articular cartilage. The tibial plateau cartilage, $2 \mathrm{~mm} \times 2 \mathrm{~mm} \times 2 \mathrm{~mm}$ in size for TEM, was fixed in $3 \%$ glutaraldehyde and $1.5 \%$ paraformaldehyde solution ( $\mathrm{pH} 7.3$ ) at $4^{\circ} \mathrm{C}$ for $24 \mathrm{~h}$, postfixed with $1 \%$ osmic acid and $1.5 \%$ potassium hexacyanoferrate (II) solution (pH 7.3) at $4^{\circ} \mathrm{C}$ for $2 \mathrm{~h}$. The samples were then washed, dehydrated with graded alcohol, and embedded in Epon-Araldite resin. Ultrathin sections were cut on a Leica ultramicrotome and stained with $2 \%$ aqueous uranyl acetate, counterstained with $0.3 \%$ lead citrate and examined with TEM (H7650, JEOL).

The subchondral bone was dried at $180^{\circ} \mathrm{C}$ for $24 \mathrm{~h}$ to grind and pass the powder through 200-mesh steel sieve, and examined with High Resolution TEM (JEM-2010, JEOL).

Western blot analysis. Protein $(20 \mu \mathrm{g})$ of each sample was heated to $100^{\circ} \mathrm{C}$ for $5 \mathrm{~min}$ and then resolved on a $10 \%$ sodium dodecyl sulfate polyacrylamide gel electrophoresis (SDS-PAGE) gel. The proteins were transferred to methanolwetted polyvinylidene difluoride (PVDF) membranes in Tris/Glycine transfer buffer. Subsequently, the membranes were blocked for $1 \mathrm{~h}$ at room temperature in blocking buffer (5\% skim milk powder, $0.5 \%$ Tween-20 in tris-buffered saline; TBS). Blots were incubated with LC3 I/II, ULK1, Beclin1 and $\beta$-actin (Abcam, Cambridge, UK) followed by an HRP-conjugated secondary antibody. Immunoreactive proteins were visualized by Western Blot Chemiluminescence Luminol Reagent (Santa Cruz Biotechnology, Santa Cruz, CA, USA). Immunoblot bands were quantitated with the Tocan 190 protein assay system (Bio-Rad, USA). 
OA group
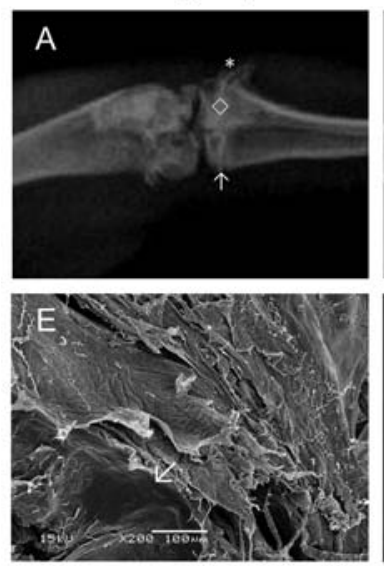

Control group
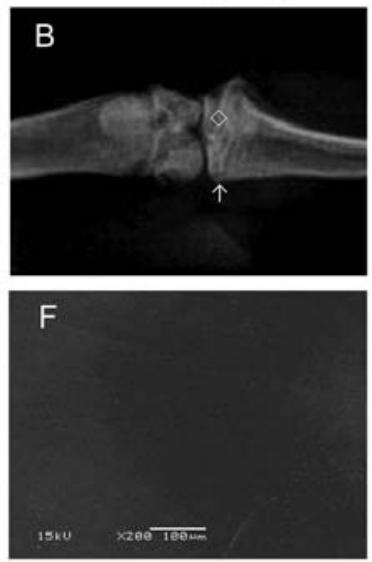

$\mathrm{GlcN}+\mathrm{OA}$ group
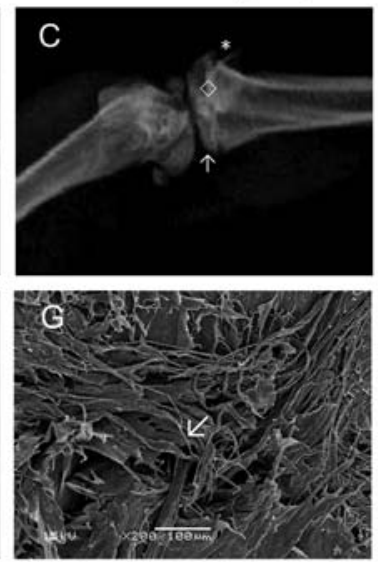

TXC+OA group
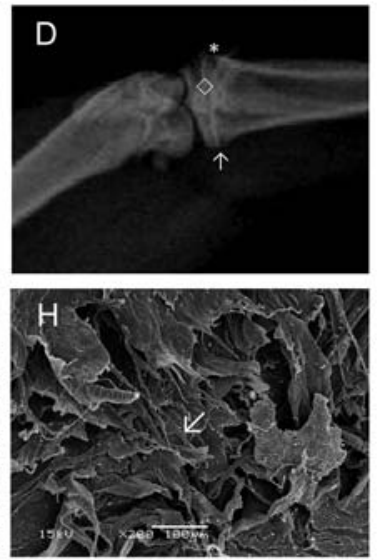

Figure 1. Morphology analyses of the knee joints treated with TXC. TXC treatment improved radiological indices of OA: X-ray results. The OA group (A); the control group (B); the GlcN+OA group (C); the TXC+OA group (D). Arrows indicate tibial plateau, diamonds indicate subchondral bone, and stars indicate osteophytes. The surface of tibial plateau obtained from SEM images of an OA knee (E), a control knee (F), a GlcN+OA knee (G) and a TXC+OA knee (H). The OA knee showed erosion and pitting of the tibial plateau, which was more severe in the medial tibial plateau (arrows). The control knee maintained the tibial plateau integrity with a smooth contour.

Statistical analysis. All data are represented as the means of averages \pm standard deviation (SD) and analyzed by using the SPSS package for Windows (version 13.0). Statistical analysis of the data was performed with Student's t-test and ANOVA. The enumeration data was analyzed by the Chi-square test. $\mathrm{P}<0.05$ was considered to indicate statistically significant differences.

\section{Results}

No signs of drug toxicity were noted in the SD rats treated with TXC or GlcN. The level of daily activity was similar in all four experimental groups, and there were no significant differences in body weight between the groups during the study period.

TXC delays the degradation of papain-induced $O A$. In the knee joints, gross morphologic changes with a significant difference between the control group and the treatment with TXC or GlcN and OA groups are shown in Fig. 1. The width of the hind limb knee joint of the TXC or GlcN group was clearer and wider than that of the OA group. In the OA group, gross morphologic changes were characterized by cartilage degradation, such as fibrillation, erosion and ulcer formation, and osteophyte formation, observed in the femoral condyle and tibial plateau. Markedly less severity of knee joint degradation was observed following treatment with TXC or GlcN (Table I; $\mathrm{P}<0.05)$.

TXC inhibits cartilage tidemark replication. Tibial plateau cartilage from the OA group showed evident histological changes, such as moderate-to-severe hypocellularity, complete disorganization, proteoglycan reduction on Safranin O-fast green staining of ECM, denudation of articular surface and fissures extending into the deep zones, and tidemark replication, compared to the control group (Fig. 2A-F). Osteophytes were present at the medial margins of the tibial plateau. In turn, tibial plateau cartilage from the control group showed homogeneous and intense staining of proteoglycans at ECM, normal cellularity and structure across the different layers,
Table I. Kellgren-Lawrence X-ray grade standard in the different groups.

\begin{tabular}{lrcccc}
\hline Group & G0 & G1 & G2 & G3 & G4 \\
\hline OA & 0 & 0 & 0 & 4 & 6 \\
Control $^{\mathrm{b}}$ & 10 & 0 & 0 & 0 & 0 \\
GlcN+OA $^{\mathrm{a}, \mathrm{c}}$ & 0 & 0 & 2 & 6 & 2 \\
TXC+OA $^{\mathrm{a}, \mathrm{c}}$ & 0 & 0 & 3 & 5 & 2
\end{tabular}

${ }^{\mathrm{a}} \mathrm{P}<0.05,{ }^{\mathrm{b}} \mathrm{P}<0.01$, compared with the $\mathrm{OA}$ group; ${ }^{\mathrm{C}} \mathrm{P}<0.01$ compared with the control group. G, Grade.

and tidemark wave-shaped (Fig. 2G and H). As shown in Fig. 2I-L, tibial plateau cartilage following the treatment with TXC or GlcN displayed improvement in cellularity and cellular organization in chondron-like manner, although there was some diffuse hypercellurarity compared to the OA group. There was also reduction of structure irregularities and mild improvement of ECM. The Mankin scores of cartilage in both the TXC or GlcN and OA groups were significantly higher than those in the control group $(\mathrm{P}<0.01)$, and in the TXC or GlcN groups they were significantly lower than those in the OA group $(\mathrm{P}<0.01)$ (Fig. $2 \mathrm{M})$.

TXC delays chondrocyte and subchondral bone degradation. Ultrastructural study of tibial plateau cartilage in both the TXC or GlcN and OA groups by TEM revealed distinctive differences compared to control cartilages. The cartilage in the OA group showed microscopic evidence of surface fibrillation, loss of ECM staining in the superficial region for proteoglycans, several apoptotic cells and reduced cellularity. Apoptotic cells were shrunken and clearly retracted from the surrounding ECM. The ultrastructural characteristics of apoptotic cells that we observed were the presence of nuclear blebbing, apoptotic bodies and cell shrinkage, whereas intensified staining of the 

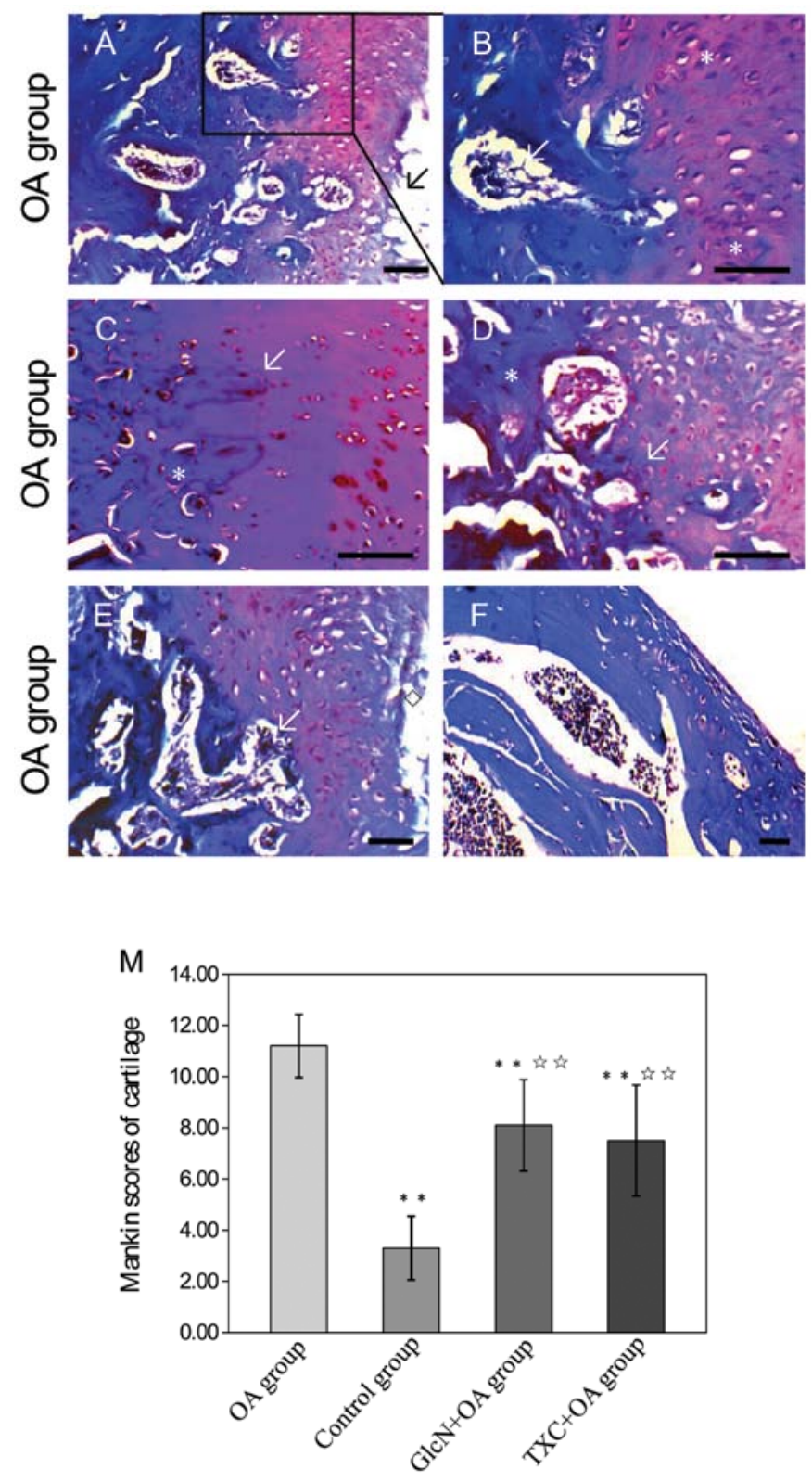

cytoplasm, blebbing of the cell membrane, and condensation of the chromatin were observed less frequently (Fig. 3A and B). The ECM contained degradation of several collagen fibers (Fig. 3C).

The subchondral bone showed loosening and irregularity of collagen arrangement, dense crystals of calcium and phosphorus (Fig. 3D). The cartilage in the control group showed chondrocytes were generally round and characterized by several microvilli-like structures and corrugations at the chondrocytic surface, some cells with the characteristics of autophagosomes (Fig. 3E). The cytoplasm contained some mitochondria and a relatively abundant number of organelles assumed either rodlike or spherical morphology, and presented clearly visible cristae. The nucleus of chondrocytes showed lobate or indentation morphology, heterochromatin was observed preferentially at the periphery of the nucleus (Fig. 3F). The ECM typically consists of a network of tightly packed and highly cross-linked
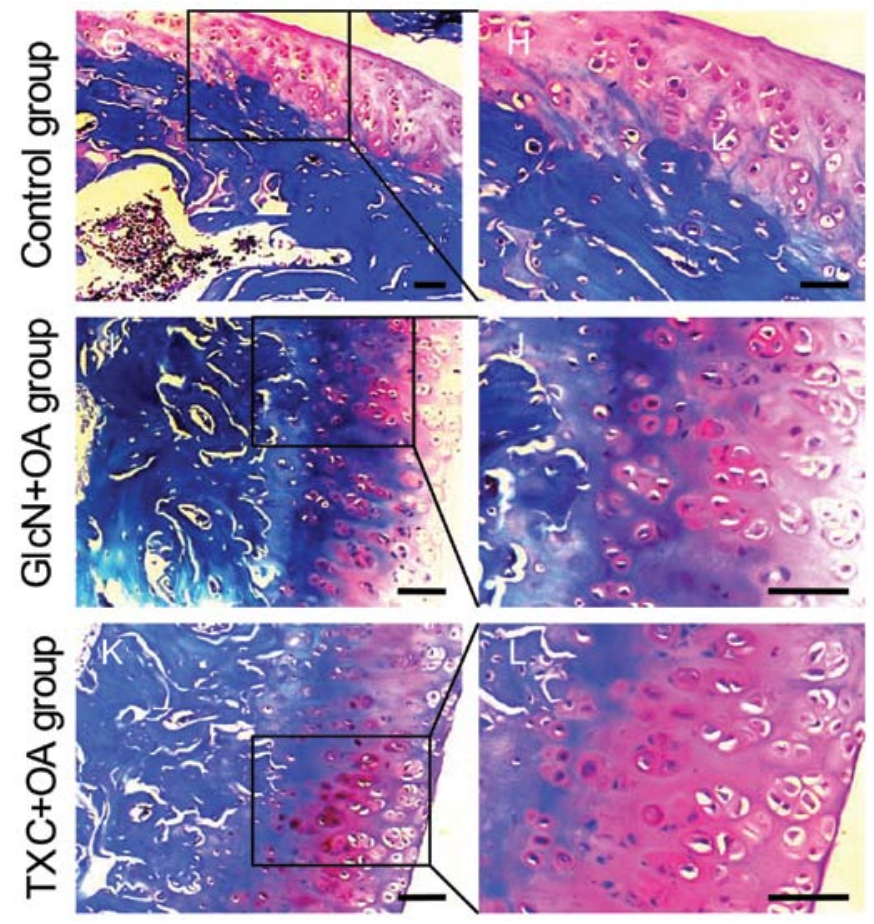

Figure 2. Histology analyses of the medial tibial plateau treated with TXC Histological sections of the tibial plateau stained with Safranin O-fast green to illustrate pathological changes. The OA tibial plateau showed loss of viable chondrocytes (A, arrow), chondrocyte proliferation (B, asterisk), loss of proteoglycans, and tidemark replication (C and $\mathrm{D}$, arrow). The cartilage showed fibrillation, vertical fissures (E, diamond) and delamination (F). The subchondral trabecular bone architecture was altered with sclerosed bone ( $\mathrm{C}$ and $\mathrm{D}$, asterisk) and the cellular bone marrow was replaced by loosely arranged spindle cells in a fine fibrous stroma (B and E, arrow). The control tibial plateau showed normal healthy cartilage with normally distributed chondrocytes $(\mathrm{G})$ and tidemark wave-shaped $(\mathrm{H}$, arrow). The red coloration of the hyaline cartilage, reflecting proteoglycan content, is homogenous and the surface of the cartilage is smooth. The GlcN+OA tibial plateau showed the cartilage is lost, the hyaline cartilage has reduced proteoglycans (loss of staining), a reduced thickness (erosion) is diffusely hypocellular and clones are present at the periphery of the changes ( $\mathrm{I}$ and $\mathrm{J}$ ). There appears to be an increase in the number and size of bone lacunae under the pits. The overlying hyaline cartilage in TXC+OA has increased proteoglycans compared to the OA cartilage (K and L). Mankin score analysis of the medial tibial plateau (M). Symbols represent the means of averages \pm SD and SD is shown as

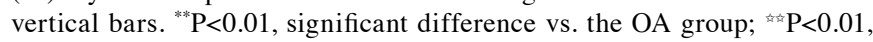
significant difference vs. the control group.

collagen fibrils (Fig. 3G). The subchondral bone showed regularity of collagen arrangement, uniform distribution of calcium phosphate crystals (Fig. 3H). Markedly less severity of cartilage and subchondral bone degradation was observed in the treatment with TXC or GlcN (Fig. 3I-Q).

TXC enhances the autophagy-related proteins against chondrocyte apoptosis. We then examined whether another type of cell death, autophagy, was involved in OA pathogenesis. We first examined the conversion of LC3 from an $18 \mathrm{kDa}$ form (LC3 I) to a faster-migrating $16 \mathrm{kDa}$ form (LC3 II), ULK1 and Beclin1. As shown in Fig. 4A-E, a western blot assay demonstrated a decrease in LC3 I/II, ULK1 and Beclin1 levels in the OA group compared to those in the control group $(\mathrm{P}<0.01)$. However, LC3 I/II, ULK1 and Beclin1 levels in both the TXC and the GlcN group significantly increased compared to those in the OA group $(\mathrm{P}<0.05, \mathrm{P}<0.01)$. 

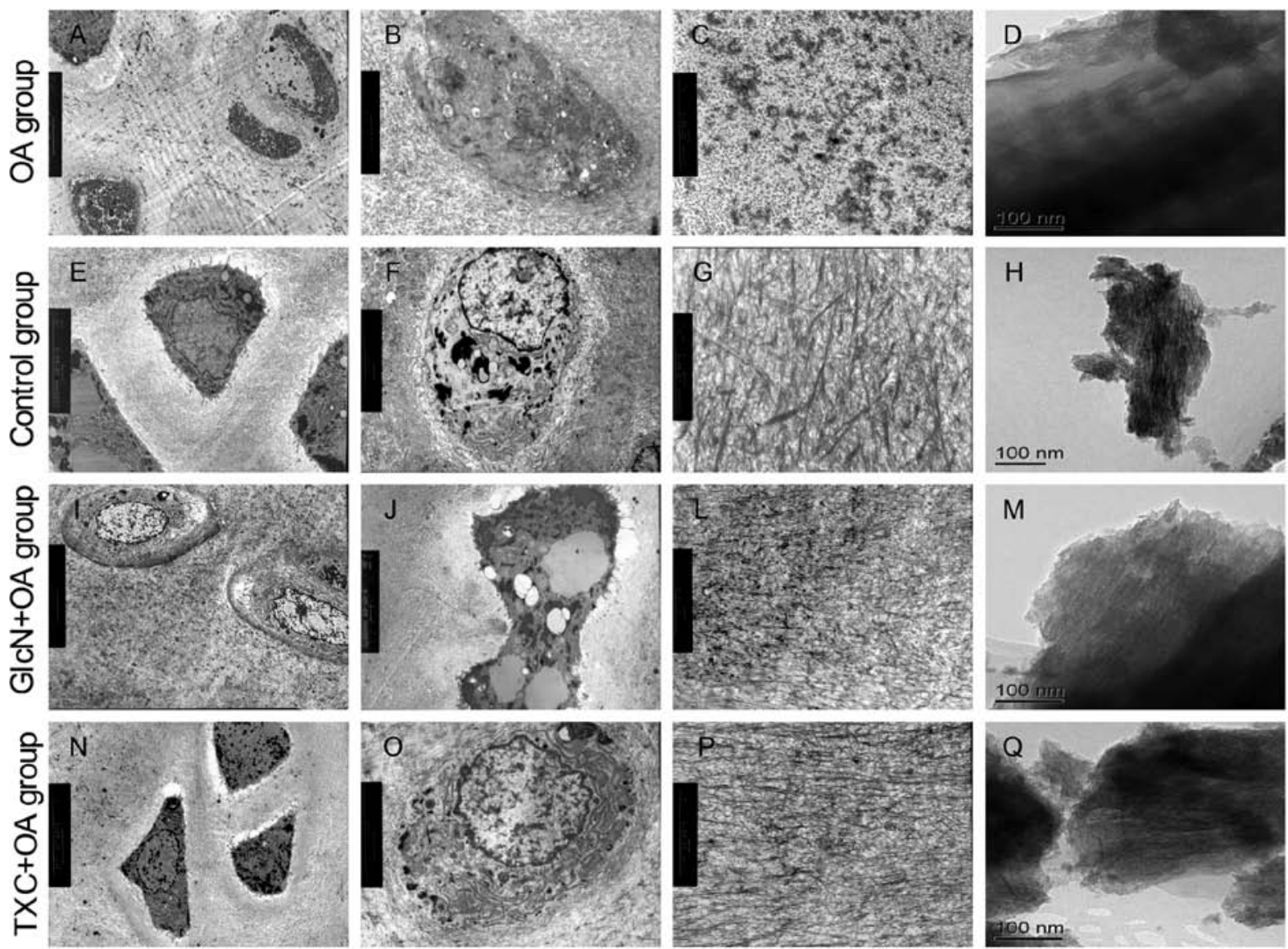

Figure 3. Analyses of ultrastructural characteristics of the tibial plateau treated with TXC. The OA tibial plateau showed chondrocytes presented nuclear blebbing, apoptotic cells, cell shrinkage (A and B), and several collagen fibers degraded (C). The subchondral bone showed loosening and irregularity of collagen arrangement, dense crystals of calcium and phosphorus (D). The control tibial plateau showed chondrocytes were generally round and characterized by numerous microvilli-like structures and corrugations at chondrocytic surface (E), some cells presented the features of autophagosomes (F). The ECM typically consists of a network of tightly packed and highly cross-linked collagen fibrils $(\mathrm{G})$. The subchondral bone showed regularity of collagen arrangement, uniform distribution of calcium phosphate crystals $(\mathrm{H})$. Markedly less severity of cartilage and subchondral bone degradation was observed in the treatment with TXC or GlcN (I-Q), showing early autophagic cells with a nucleus and microvilli, such as several autophagic vacuoles in the cytoplasm, large vacuoles approaching mitochondria and membrane whorls.

\section{Discussion}

The present study systematically evaluated the cartilage protection mechanisms of TXC in papain-induced OA. Our results clearly showed that TXC could improve the arrangement of subchondral bone collagen fibers and calcium phosphate crystals, inhibit the tidemark replication and delay the cartilage degradation. In addition, our study showed that TXC upregulated the protein levels of LC3 I/II, ULK1 and Beclin1, demonstrating that it could inhibit the tidemark replication and cartilage degradation by the activation of chondrocyte autophagy.

A number of treatment programs of OA have been developed, such as medications with NSAIDs and chondroprotective drugs. However, major problems associated with medications still remain, particularly with the side-effects of NSAIDs. Thus, there is a pressing need to develop alternative approaches to OA treatment (17). Complementary and alternative medicine is currently of interest to the general public and the medical profession, and includes TCM. Chinese herbs have relatively fewer-side effects compared to modern chemotherapeutics and have long been used clinically to treat OA. TXC, a famous
TCM formulation, has been reported to be clinically effective in treating OA by inhibiting chondrocyte apoptosis (14). However, the molecular mechanism of the therapeutic effect of TXC remains largely unknown. Therefore, the present study examined whether TXC regulates the tidemark replication and cartilage degradation by the regulation of chondrocyte autophagy.

Tidemark, a distinct boundary between non-calcified and calcified articular cartilage and not an artifact, has been described as a haematoxyphil single line which is approximately $10-\mu \mathrm{m}$ thick $(18,19)$. Chondrocytes near the tidemark must regulate the turnover of non-collagenous components in ECM and maintain control over the local ECM (20). During normal development and growth of diarthrodial joints, the tidemark clearly represents a calcification front. In the normal adult joint, the tidemark is still a single structure, ceases the advance of mineral into the hyaline cartilage, although a residual 'maintenance' turnover of ECM may occur (21). Under these conditions, although the tidemark still contains some tightly bound calcium, it may have ceased to function as a calcification front. It is possible that the tidemark has changed in function to one of inhibiting the growth or forma- 

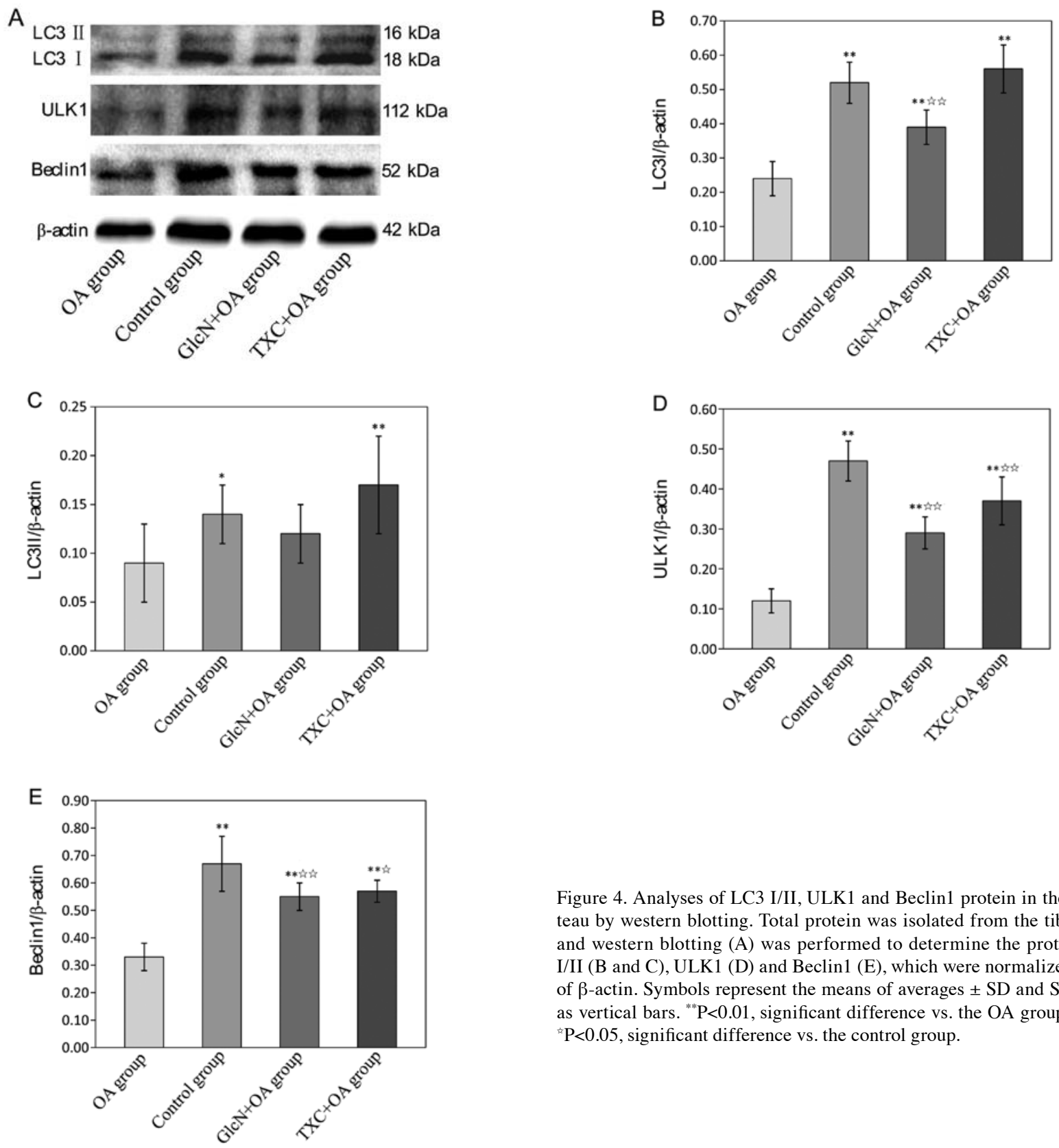

Figure 4. Analyses of LC3 I/II, ULK1 and Beclin1 protein in the tibial plateau by western blotting. Total protein was isolated from the tibial plateau and western blotting (A) was performed to determine the protein of LC3 I/II (B and C), ULK1 (D) and Beclin1 (E), which were normalized with that of $\beta$-actin. Symbols represent the means of averages \pm SD and SD is shown as vertical bars. ${ }^{* *} \mathrm{P}<0.01$, significant difference vs. the $\mathrm{OA}$ group; ${ }^{\text {齐 }} \mathrm{P}<0.01$, ${ }^{\circledR} \mathrm{P}<0.05$, significant difference vs. the control group.

tion of microcrystals of hydroxyapatite result in protecting the hyaline cartilage from passive progressive mineralization at this stage. This may be an irreversible change, so that if new mineralization is activated in OA, a new tidemark may actively form distally to the original one, leaving its predecessor as a non-functional relic, and thus providing an explanation of tidemark replication. The tidemark replication in OA is characterized by an endochondral ossification process advancing calcification of zone IV and replacement of calcified cartilage by new bone at the calcified cartilage-bone interface, and these events must be precisely determined and regulated by adjacent chondrocytes (22). Histological measures of articular cartilage pathology, generally considered to be the reference standard for presence and severity of OA, showed significant tidemark replication and cartilage degradation in the OA group compared to the control group, indicating that OA was successfully induced by papain. In the present study, the
TXC+OA group showed a smaller increase in knee joint width and an inhibition of cartilage degradation as compared to the OA group. Collectively, the above findings support a role for TXC in the protection of cartilage and chondrocyte metabolism, suggesting a possible mechanism by which TXC may help to alleviate clinical signs and retard progression of OA.

$\mathrm{OA}$ is a characterized by cartilage degradation and subchondral bone changes. Microcracks in the calcified tissues may enhance cellular activity leading to increased bone remodeling $(23,24)$. Although the relationship between cartilage degradation and subchondral bone changes remains controversial, the subchondral bone is considered to play an important role in OA initiation and progression. During the OA process, the subchondral bone would then become stiffer, causing a reduced shock-absorbing capacity and leading to progression of these lesions $(25,26)$. However, other research shows that the stiffness of subchondral bone in OA is actually 
decreased, due to a reduced mineral content and an increased porosity (27). Further studies have shown the phenomenon to be more complex and to involve changes in collagen content and bone density, which potentially lead to weakened subchondral trabecular bone $(28,29)$. We found that the subchondral bone degradation of the TXC+OA group significantly decreased compared to the OA group, indicating the regulation of tidemark replication by affecting the arrangement of subchondral bone collagen fibers and calcium phosphate crystals is one of the mechanisms by which TXC may be effective in the treatment of OA.

Cell death can be classified according to enzymological criteria, morphological appearance, immunological characteristics or functional aspects. Based on morphological criteria, three types of cell death can be defined; necrosis, apoptosis and autophagy. Autophagy is a lysosomal degradation pathway that is essential for differentiation, survival, homeostasis and development. However, in certain physiological and pathological conditions, autophagy can also result in a form of cell death that is termed type II programmed cell death; the morphological hallmark of autophagy forms the sequestering vesicle, the autophagosome, which fuses with lysosomes and degrades and recycles cellular components (30). Atg genes regulate the autophagy process resulting in the induction and nucleation of autophagic vesicles, their expansion and fusion with lysosomes, and the enzymatic degradation and recycling (31,32). Among the Atg genes, Atg1, Atg5, Atg6 and Atg8 (ULK1, Beclin1 and LC3 in mammals, respectively) are four major regulators of the autophagy pathway. ULK1 is an important intermediate in the transduction of pro-autophagic signals to the formation of autophagosomes (33). Beclin1 forms a complex with Vps34 and type III PI3 kinase, and then allows nucleation of the autophagic vesicle $(34,35)$. Finally, the formation and expansion of autophagosome demands two protein conjugation systems including Atg12 and Atg proteins LC3 (36).

LC3 is the existence of two forms, LC3 II bound to the autophagosome membrane and LC3 I in the cytoplasm. During the autophagy process, LC3 I is converted to LC3 II through lipidation by a ubiquitin-like system leading to the association of LC3 II with autophagy vesicles. Therefore, the amount of LC3 II is correlated with the extent of autophagosome formation $(12,37)$. The present results showed that autophagy was decreased in papain-induced OA, and the feature of papaininduced cartilage degradation increased cell death suggesting that loss of autophagy may contribute to cell death. Our results also showed that the protein of LC3 I/II, ULK1 and Beclin1 in osteoarthritic cartilage was significantly decreased, and TXC could increase these autophagy markers, demonstrating activation of autophagy is one of the mechanisms by which TXC inhibits the papain-induced cartilage degradation.

In summary, the protective role of autophagy in endochondral ossification was further supported by observations that its inactivation leads to severe skeletal abnormalities, due in part to cell death (38). This study confirmed that autophagy may be a protective or homeostatic mechanism in normal cartilage. TXC could enhance chondrocyte autophagy by promoting the expression of LC3 I/II, ULK1 and Beclin1 to inhibit the tidemark replication and cartilage degradation in the papaininduced $\mathrm{OA}$. These results suggested that compromised autophagy may represent a novel avenue to delay the development of OA. Since autophagy can serve to delay the onset of apoptosis, further experiments are in progress to explore the mechanism of TXC in the regulation of the relationship between the induction of autophagy and apoptosis.

\section{Acknowledgements}

This study was supported by the National Natural Science Foundation of China (Grant no. 81102609), the Key Project of Fujian Provincial Department of Science and Technology (Grant no. 2012Y0046), the Natural Science Foundation of Fujian Province (Grant no. 2011J05074) and the Young Talent Scientific Research Project of Fujian Province Universities (Grant no. JA12165).

\section{References}

1. Li X, Ye H, Yu F, et al: Millimeter wave treatment promotes chondrocyte proliferation via $\mathrm{G}_{1} / \mathrm{S}$ cell cycle transition. Int $\mathbf{J} \mathrm{Mol}$ Med 29: 823-831, 2012.

2. Wu MX, Li XH, Lin MN, et al: Clinical study on the treatment of knee osteoarthritis of shensui insufficiency syndrome type by electroacupuncture. Chin J Integr Med 16: 291-297, 2010.

3. Galois L, Etienne S, Grossin L, et al: Dose-response relationship for exercise on severity of experimental osteoarthritis in rats: a pilot study. Osteoarthritis Cartilage 12: 779-786, 2004.

4. Panicker S, Borgia J, Fhied C, Mikecz K and Oegema TR: Oral glucosamine modulates the response of the liver and lymphocytes of the mesenteric lymph nodes in a papain-induced model of joint damage and repair. Osteoarthritis Cartilage 17: 1014-1021, 2009.

5. Eswaramoorthy R, Chang CC, Wu SC, Wang GJ, Chang JK and Ho ML: Sustained release of PTH(1-34) from PLGA microspheres suppresses osteoarthritis progression in rats. Acta Biomater 8: 2254-2262, 2012.

6. Bonde HV, Talman ML and Kofoed H: The area of the tidemark in osteoarthritis - a three-dimensional stereological study in 21 patients. APMIS 113: 349-352, 2005.

7. Lyons TJ, Stoddart RW, McClure SF and McClure J: The tidemark of the chondro-osseous junction of the normal human knee joint. J Mol Histol 36: 207-215, 2005.

8. Dequeker J, Mokassa L, Aerssens J and Boonen S: Bone density and local growth factors in generalized osteoarthritis. Microsc Res Tech 37: 358-371, 1997.

9. Del Carlo M Jr and Loeser RF: Cell death in osteoarthritis. Curr Rheumatol Rep 10: 37-42, 2008.

10. Almonte-Becerril M, Navarro-Garcia F, Gonzalez-Robles A, Vega-Lopez MA, Lavalle C and Kouri JB: Cell death of chondrocytes is a combination between apoptosis and autophagy during the pathogenesis of Osteoarthritis within an experimental model. Apoptosis 15: 631-638, 2010.

11. Kim HA, Lee YJ, Seong SC, Choe KW and Song YW: Apoptotic chondrocyte death in human osteoarthritis. J Rheumatol 27: 455-462, 2000.

12. Caramés B, Taniguchi N, Otsuki S, Blanco FJ and Lotz M: Autophagy is a protective mechanism in normal cartilage, and its aging-related loss is linked with cell death and osteoarthritis. Arthritis Rheum 62: 791-801, 2010.

13. Lotz MK and Caramés B: Autophagy and cartilage homeostasis mechanisms in joint health, aging and OA. Nat Rev Rheumatol 7: 579-587, 2011.

14. Li XH, Wu MX, Ye HZ, et al: Experimental study on the suppression of sodium nitroprussiate-induced chondrocyte apoptosis by Tougu Xiaotong Capsule-containing serum. Chin J Integr Med 17: 436-443, 2011.

15. Kellgren JH and Lawrence JS: Radiological assessment of rheumatoid arthritis. Ann Rheheum Dis 16: 485-493, 1957.

16. Mankin HJ, Dorfman H, Lippiello L and Zarins A: Biochemical and metabolic abnormalities in articular cartilage from osteoarthritic human hips. II. Correlation of morphology with biochemical and metabolic data. J Bone Joint Surg Am 53: 523-537, 1971.

17. Klop C, de Vries F, Lalmohamed A, et al: COX-2-selective NSAIDs and risk of hip or knee replacements: a populationbased case-control study. Calcif Tissue Int 91: 387-394, 2012. 
18. Zoeger N, Roschger P, Hofstaetter JG, et al: Lead accumulation in tidemark of articular cartilage. Osteoarthritis Cartilage 14: 906-913, 2006

19. Gannon FH and Sokoloff L: Histomorphometry of the aging human patella: histologic criteria and controls. Osteoarthritis Cartilage 7: 173-181, 1999.

20. Meirer F, Pemmer B, Pepponi G, et al: Assessment of chemical species of lead accumulated in tidemarks of human articular cartilage by X-ray absorption near-edge structure analysis. J Synchrotron Radiat 18: 238-244, 2011.

21. Otterness IG, Chang M, Burkhardt JE, Sweeney FJ and Milici AJ: Histology and tissue chemistry of tidemark separation in hamsters. Vet Pathol 36: 138-145, 1999.

22. Pan J, Wang B, Li W, et al: Elevated cross-talk between subchondral bone and cartilage in osteoarthritic joints. Bone 51: 212-217, 2012

23. Lacourt M, Gao C, Li A, et al: Relationship between cartilage and subchondral bone lesions in repetitive impact traumainduced equine osteoarthritis. Osteoarthritis Cartilage 20: 572-583, 2012.

24. Bobinac D, Spanjol J,Zoricic S and Maric I: Changes in articular cartilage and subchondral bone histomorphometry in osteoarthritic knee joints in humans. Bone 32: 284-290, 2003.

25. Burr DB: Increased biological activity of subchondral mineralized tissues underlies the progressive deterioration of articular cartilage in osteoarthritis. J Rheumatol 32: 1156-1158, 2005.

26. Botter SM, Glasson SS, Hopkins B, et al: ADAMTS5-/- mice have less subchondral bone changes after induction of osteoarthritis through surgical instability: implications for a link between cartilage and subchondral bone changes. Osteoarthritis Cartilage 17: 636-645, 2009.

27. Day JS, Ding M, van der Linden JC, Hvid I, Sumner DR and Weinans H: A decreased subchondral trabecular bone tissue elastic modulus is associated with pre-arthritic cartilage damage. J Orthop Res 19: 914-918, 2001.
28. Bennell KL, Creaby MW, Wrigley TV and Hunter DJ: Tibial subchondral trabecular volumetric bone density in medial knee joint osteoarthritis using peripheral quantitative computed tomography technology. Arthritis Rheum 58: 2776-2785, 2008.

29. Aula AS, Töyräs J, Tiitu V and Jurvelin JS: Simultaneous ultrasound measurement of articular cartilage and subchondral bone. Osteoarthritis Cartilage 18: 1570-1576, 2010.

30. Mizushima N, Levine B, Cuervo AM and Klionsky DJ: Autophagy fights disease through cellular self-digestion. Nature 451: 1069-1075, 2008.

31. Srinivas V, Bohensky J, Zahm AM and Shapiro IM: Autophagy in mineralizing tissues: microenvironmental perspectives. Cell Cycle 8: 391-393, 2009.

32. Cajee UF, Hull R and Ntwasa M: Modification by ubiquitin-like proteins: significance in apoptosis and autophagy pathways. Int $\mathbf{J}$ Mol Sci 13: 11804-11831, 2012.

33. Chan EY, Kir S and Tooze SA: siRNA screening of the kinome identifies ULK1 as a multidomain modulator of autophagy. J Biol Chem 282: 25464-25474, 2007.

34. Furuya N, Yu J, Byfield M, Pattingre S and Levine B: The evolutionarily conserved domain of Beclin 1 is required for Vps34 binding, autophagy and tumor suppressor function. Autophagy 1: 46-52, 2005 .

35. Abounit K, Scarabelli TM and McCauley RB: Autophagy in mammalian cells. World J Biol Chem 3: 1-6, 2012

36. Ohsumi Y and Mizushima N: Two ubiquitin-like conjugation systems essential for autophagy. Semin Cell Dev Biol 15: 231-236, 2004.

37. Kabeya Y, Mizushima N, Ueno T, et al: LC3, a mammalian homologue of yeast Apg8p, is localized in autophagosome membranes after processing. EMBO J 19: 5720-5728, 2000.

38. Settembre C, Arteaga-Solis E, McKee MD, et al: Proteoglycan desulfation determines the efficiency of chondrocyte autophagy and the extent of FGF signaling during endochondral ossification. Genes Dev 22: 2645-2650, 2008. 